\title{
El desconocido autogiro ambulancia de 1933 , uma innovación española predecesora en una década a la primera utilización del helicóptero sanitario
}

\author{
González Canomanuel MA. ${ }^{1}$
}

Sanid. mil. 2015; 71 (2): 125-131; ISSN: 1887-8571

\section{RESUMEN}

El helicóptero sanitario ha revolucionado el transporte de enfermos. Es el único medio de evacuación que lleva la atención sanitaria donde se encuentra el enfermo, trasladándolo de forma rápida y cómoda sin depender de vías de comunicación o instalaciones. La participación española en la creación de las "aeronaves de ala rotatoria" para uso sanitario, a la que pertenece el helicóptero, es desconocida por la inmensa mayoría de profesionales que trabajan en este sector no habiendo sido nunca publicada. En 1920 el español Juan de la Cierva y Codorniú inventó el "autogiro" revolucionando la forma de volar y resolviendo los problemas del vuelo a baja velocidad. Este aparato solucionaba la limitación que tenían los aviones sanitarios de ala fija para recoger heridos por la necesidad de un campo preparado para el despegue o aterrizaje. Fueron médicos, ingenieros y aviadores españoles los que se dieron cuenta de las posibilidades en la aplicación sanitaria del autogiro promoviendo su uso en múltiples foros desde 1929. El propio Juan de la Cierva pensó en la aplicación sanitaria de su invento y este se utilizó ya en los años 30 para este fin. El proyecto del autogiro ambulancia data del año 1933 describiendo la modificación sanitaria de un autogiro de cuatro plazas PA-19 de la Pitcairn Autogiro Company. La innovación española del autogiro sanitario adelanta en más de una década a la admitida primera utilización de una aeronave de ala rotatoria para uso sanitario en el año 1944.

PALABRAS CLAVE: Transporte aéreo sanitario, helitransporte, evacuación aérea

The stranger autogiro ambulance 1933, a Spanish innovation predecessor in a decade to the first use of medical helicopter SUMMARY: The medical helicopter has revolutionized patient transport. It is the only means of escape leading healthcare where the sick, transferring it to quickly and easily without relying on roads or facilities. The Spanish participation in the creation of "rotary wing aircraft" for sanitary use, which the helicopter belongs, is unknown to the vast majority of professionals working in this sector never having been published. In 1920 the Spanish Juan de la Cierva and Codorniú invented the "autogiro" revolutionizing the way to fly and solving the problems of low speed flight. This device solved the limitation that health had fixed wing aircraft to pick up wounded by the need of a field ready for takeoff or landing. They were doctors, engineers and Spanish aviators who realized the possibilities in healthcare application of autogiro promoting their use in multiple forums since 1929. Juan de la Cierva own thought in health application of his invention and it is already used in 30 years for this purpose. The project gyroplane ambulance dates from 1933 describing the health modifying a four-seat gyroplane PA-19 Pitcairn Autogiro Company. The Spanish healthcare innovation autogyro forward in more than a decade to taken first use of a rotary wing aircraft for domestic use in 1944.

KEY WORDS: Air medical transport, Helicopter transport, Aeromedical evacuation.

\section{INTRODUCCIÓN}

El uso de aviones para el transporte sanitario fue una idea rápidamente aplicada desde el nacimiento de la aviación. El conflicto bélico de la I Guerra Mundial fue el escenario donde comenzó este nuevo tipo de transporte con el traslado de los primeros heridos ${ }^{1}$.

La necesidad de utilización de campos de aviación preparados para el despegue y aterrizaje de estos aparatos fue un reque-

${ }^{1}$ Adjunto del Servicio de Urgencias. Hospital Virgen de la Salud. Toledo. España.

Dirección para correspondencia: Miguel Ángel González Canomanuel. Adjunto del Servicio de Urgencias. Hospital Virgen de la Salud, Av. de Barber nº 30, C.P. 45071 Toledo. Email: magycm10@yahoo.es

Recibido: 30 de septiembre de 2014.

Aceptado: 4 de marzo de 2015. rimiento técnico que impidió que los heridos se transportasen desde posiciones lo más cercanas del frente hasta las puertas de los hospitales donde se evacuaban.

El desarrollo al final de los años 20 de pequeños aeroplanos en versión sanitaria con capacidad para transportar a un herido, como el De Havilland DH9 inglés, el Hanriot 14 y el Dorand AR franceses o el Avro 504 español, no solucionaban este problema, ya que aunque necesitaban poca pista de despegue y aterrizaje, esta se podía medir por cientos de metros ${ }^{2,3}$.

El problema que impedía que el avión pudiese despegar o aterrizar en una distancia pequeña era la necesidad de proporcionar al ala una velocidad mínima de despegue (velocidad relativa que impulsa a la aeronave al desarrollo del vuelo). Los problemas del vuelo cerca de la velocidad de perdida (la velocidad mínima a la que una aeronave es capaz de volar) se convirtieron en un reto técnico en estos primeros años de la aeronáutica. 
En el año 1920 un inventor español, Juan de la Cierva y Codorniú solucionó el problema del vuelo a baja velocidad con el desarrollo de un aparato volador completamente nuevo que patentó y denominó "Autogiro". Esta era una aeronave de ala giratoria que se propulsa mediante una hélice, al igual que los aviones, pero en vez de planos utiliza un rotor como el helicóptero. Este rotor, que gira de forma libre impulsado por el aire, genera la sustentación del aparato ${ }^{4,5}$.

Aunque parecidos, la diferencia entre un autogiro y un helicóptero estriba en que las alas en forma de aspa, "el rotor principal", que ambos tienen sobre el fuselaje, en el autogiro se mueven libremente tomando impulso gracias al aire que incide sobre ellas por el avance y proporcionando la sustentación. En el caso del helicóptero, el rotor esta unido al eje del motor que le hace girar a gran velocidad creando sustentación, con un flujo de aire inverso (de arriba hacia abajo), (Figura 1). La sencillez mecánica del autogiro es infinitamente mayor, tiene un vuelo mas "suave", tiene menos vibraciones y es mucho menos propenso aún fallo mecánico que el helicóptero, estas razones entre otras, le hacen ser un aparato idóneo para el transporte sanitario. Basta decir que ante un fallo del motor en un helicóptero, el piloto maniobra el rotor para hacer que el aparato vuele como un autogiro y así poderse salvar de un accidente catastrófico.

El helicóptero es la aeronave de ala rotatoria más conocida y utilizada en la actualidad. Su empleo en el ámbito sanitario
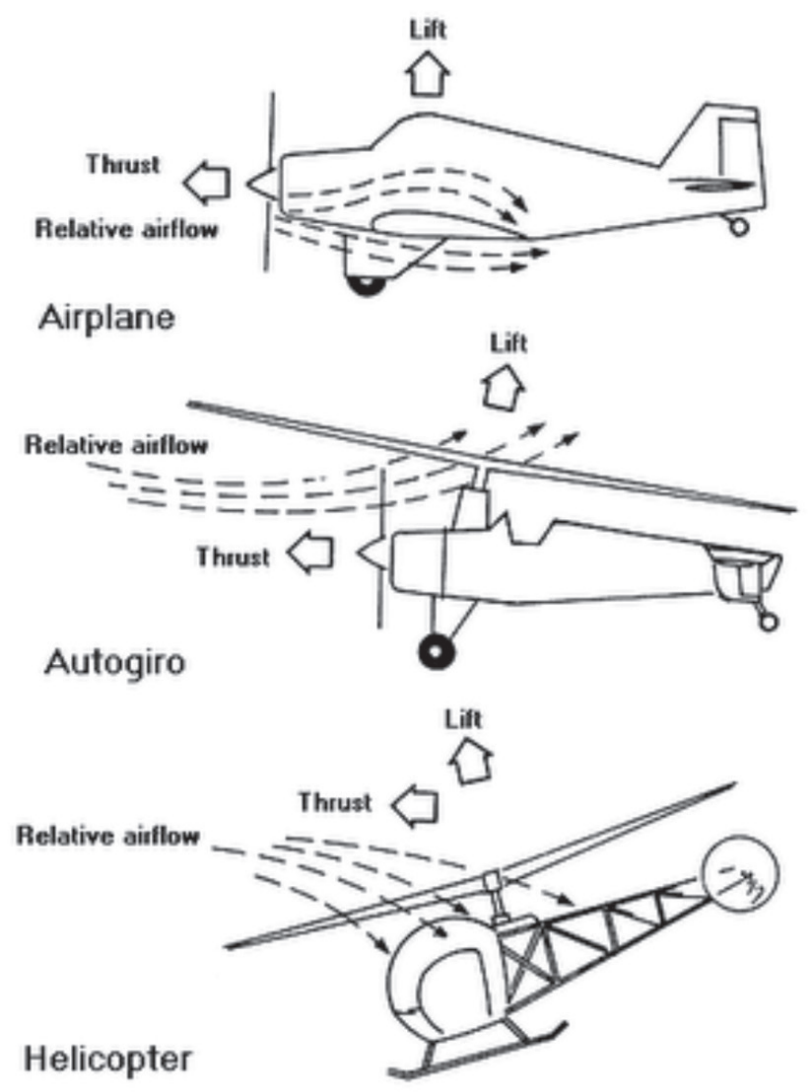

Figura 1. El autogiro tiene una forma diferente de obtener sustentación necesaria para volar que le diferencia de los aviones y de los helicópteros. La característica fundamental es la sencillez técnica y la mayor seguridad de su vuelo a baja velocidad. Imagen: www. modelismo cubano.com

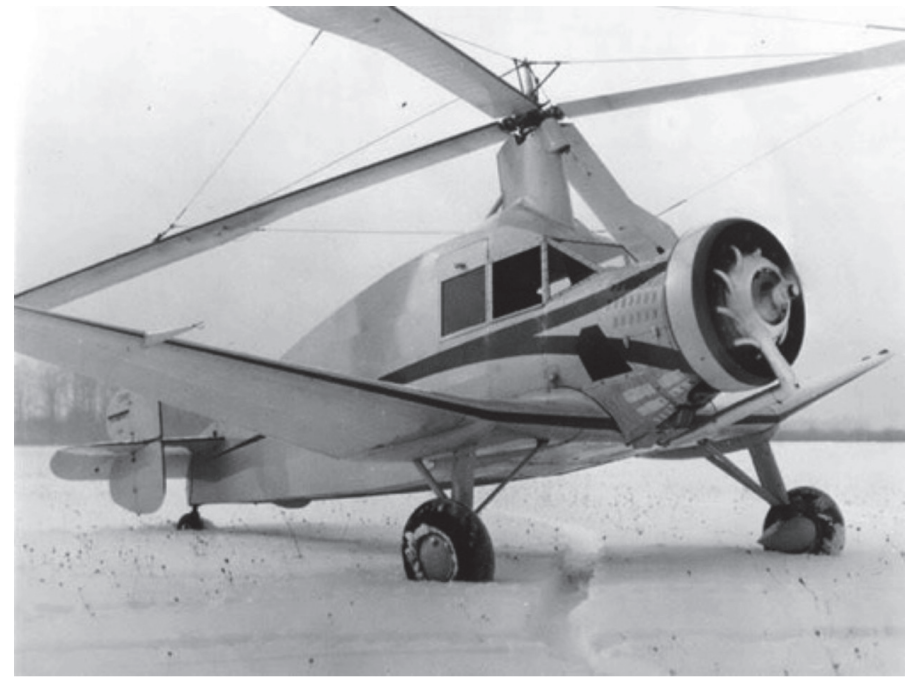

Figura 2. El modelo PA-19 fue en primer autogiro con cabina cerrada. Tenia una un nuevo sistema de movimiento manual del eje para ajuste del rotor que le proporcionaba un vuelo muy estable. Imagen colección Stephen Pitcairn.

ha supuesto una revolución en el transporte sanitario. El helicóptero moderno, desarrollado por Sikorsky, utiliza los estudios aerodinámicos y soluciones mecánicas del primer aparato de "ala rotatoria" denominado "autogiro". Siguiendo el desarrollo de los estudios del autogiro español, los alemanes al final de la segunda guerra mundial, fabrican experimentalmente el primer helicóptero operativo "Flettner F1 282". Posteriormente en 1942, un emigrante ruso en Estados Unidos, Igor Sikorsky, desarrolla y produce comercialmente el helicóptero moderno. Es en enero de 1943 cuando posteriormente a los experimentos con el prototipo XR-4, se aprueba por parte del Ejército estadounidense la producción en serie del R-4. Este será el primer helicóptero producido en cadena que, durante el final de la segunda guerra mundial en 1944, realizará la primera evacuación sanitaria en las selvas de Birmania.

La utilización de "aparatos de ala rotatoria" para uso sanitario ha sido una idea repetidamente defendida por diferentes españoles y extranjeros desde 1929 tanto en foros nacionales como internacionales. Desde la primera ponencia del I Congreso Internacional de Aviación Sanitaria celebrado en 1929 hasta en múltiples reuniones científicas posteriores. El propio inventor del autogiro Juan de la Cierva pretendió desarrollar una versión sanitaria de su invento ${ }^{5-7}$. Esta versión sanitaria se proyectará en 1933 por la empresa "Pitcairn Autogiro Company" de su modelo PA-19. Esta utiliza las patentes que posee el español Juan de La Cierva del autogiro en la "Autogiro Company of America" concesionaria para Estados Unidos de todas las patentes del autogiro español (Figura 2 y 3 ).

\section{El autogiro de Juan de la Cierva precursor del helicóptero moderno}

El invento de la aeronave de ala rotatoria lo realiza el insigne inventor español Juan de la Cierva y Codorníu en 1920, patentándolo, y realizando más de 70 modelos junto con 40 aparatos 


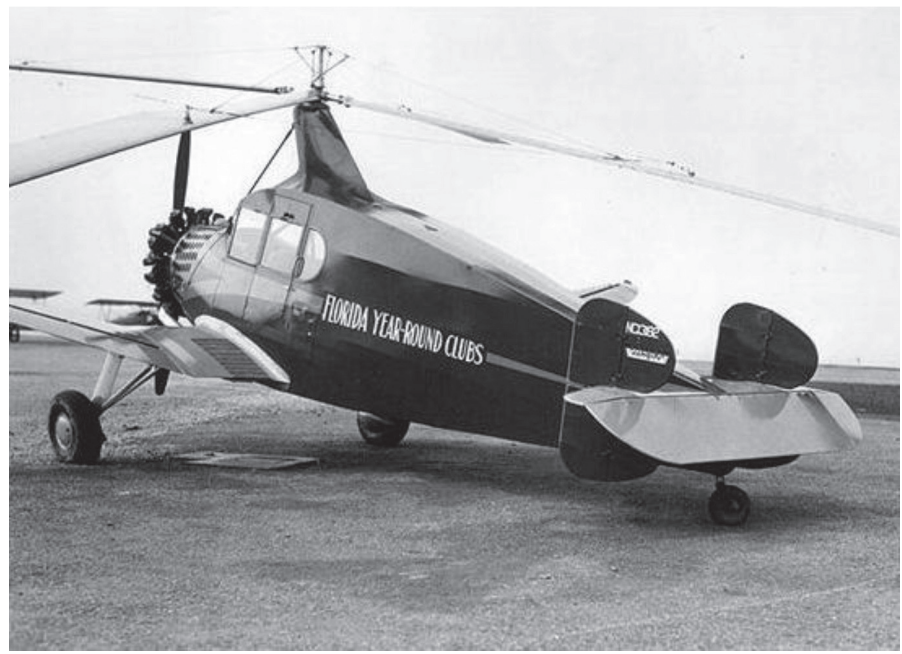

Figura 3. El modelo PA-19 era el autogiro más grande construido, la adaptación sanitaria era sencilla al poderse realizar una puerta para camillas en este lateral del fuselaje. Imagen colección Bruce H. Charnov

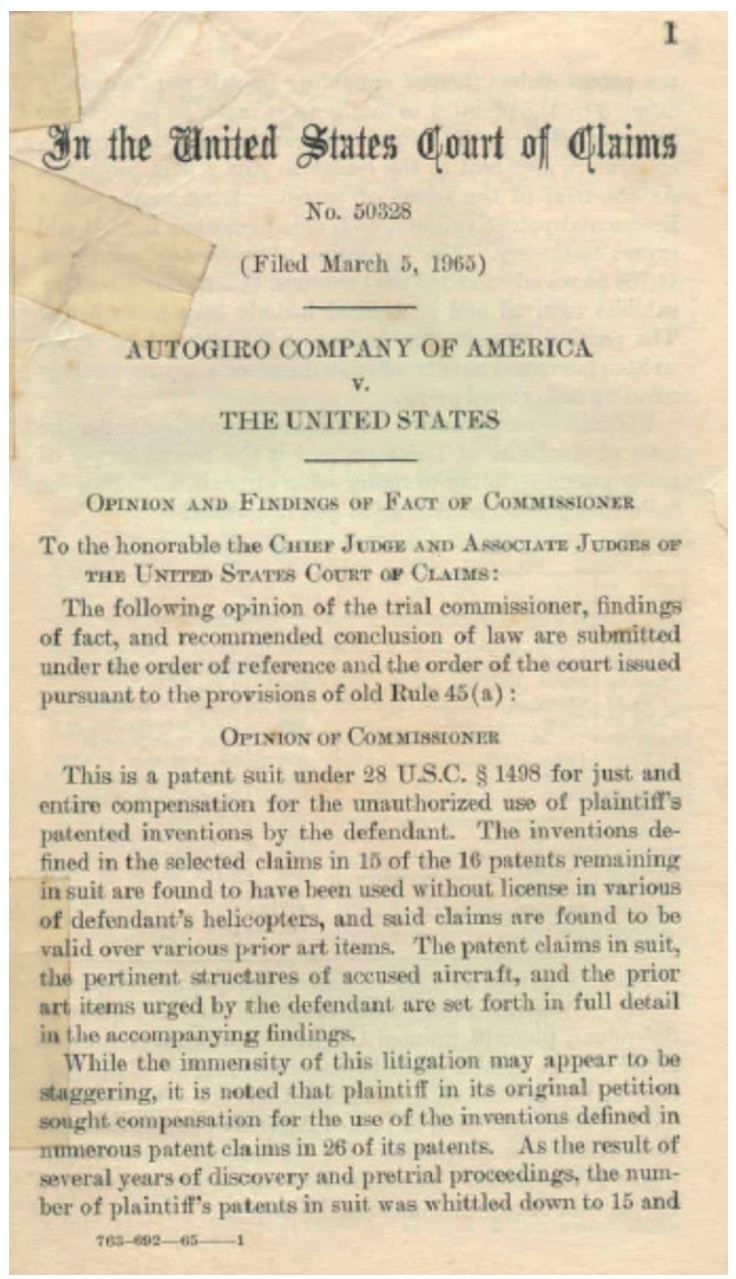

Figura 4. Portada del texto del Juez Lane donde se reconoce legalmente la utilización sin autorización de las patentes de Juan de la Cierva para el desarrollo de helicópteros. Imagen: Juan de la Cierva y Codorníu, ensayo para la fundación INTEGRA. en los siguientes 16 años antes de su prematura muerte. Aunque hubo intentos anteriores, estos tuvieron un nulo éxito. Es el autogiro de la Cierva el que logra un vuelo estable y efectivo junto con un desarrollo comercial de más de 400 aparatos fabricados en todo el mundo ${ }^{5}$. La Aviación Militar proporciona fondos y ayuda decisiva en los primeros años del desarrollo del autogiro. Juan de la Cierva soluciona el vuelo a poca velocidad con el invento de un aparato completamente nuevo así como unas soluciones mecánicas que son las que hoy en día permiten el vuelo de todos los aparatos de ala rotatoria. También desarrolla " $\mathrm{La}$ teoría aerodinámica de las alas giratorias" donde se da la explicación físico-matemática de esta nueva forma de volar. Estos nuevos inventos realizados por la Cierva como la articulación de batimiento, la articulación de arrastre, la autorrotación, el mando directo, junto con su teoría aerodinámica, son los que posibilitan el vuelo de los actuales helicópteros. Tanto es así que el padre del helicóptero moderno Igor Sikorsky, se refería a Juan de la Cierva como "inventor" del helicóptero, siendo su empresa una de las que pago por el uso de estas patentes ${ }^{4,5}$.

En 1976 el Tribunal de Apelaciones de Estados Unidos de América dicta una histórica sentencia condenando subsidiariamente al gobierno de los Estados Unidos a pagar la mayor indemnización por el uso, sin autorización, de las patentes del autogiro de la empresa Autogiro Company of América. El juez Lane hace responsable al gobierno de los EEUU por la adquisición de helicópteros de las empresas Bell, Hiller, Vertol sin las licencias de la Autogiro Company of America. No así a la principal fabricante de helicópteros, Sikorsky Aircraft, ya que esta si reconoció y pagó por la utilización de dichas patentes. La "Decisión Lane" del Tribunal de Apelaciones norteamericano prueba irrefutablemente que el helicóptero es un descendiente directo del autogiro, y que no puede existir ni volar sin usar las patentes y teorías de Juan de la Cierva ${ }^{4}$ (Figura 4).

\section{La propuesta del autogiro para labores sanitarias}

La aparición del autogiro coincide, en sus fechas, con el comienzo del desarrollo de la aviación sanitaria. En estos primeros años hay un gran entusiasmo en la organización de este nuevo campo tanto en sus aspectos legales, estructurales, sanitarios así como en los aeronáuticos. Dentro de este último, se busca la aeronave que mejor cualidades técnicas posea para el transporte sanitario $^{(1)}$. La mayoría de los países presentan internacionalmente distintas aeronaves diseñadas para este nuevo tipo de transporte aéreo como demostración de la naciente industria aeronáutica de cada uno de ellos. En el caso de España la invención por Juan de la Cierva de un nuevo tipo de aeronave distinta en su forma de volar a las que existían, ha producido gran expectación en el mundo aeronáutico internacional. Es el propio inventor del autogiro el que propone su utilización como aeronave para transporte sanitario debido a sus grandes prestaciones para este campo exponiéndolas en múltiples conferencias ${ }^{5-7}$.

(1) Incluso se crea un premio internacional la copa "Copa Maurice Ráphale" destinada al mejor avión sanitario o el avión con las mejores condiciones para el transporte de enfermos y material sanitario. 
En estos años se realiza en las primeras reuniones científicas internacionales sobre aviación sanitaria donde son médicos militares españoles y extranjeros los que participan y defienden al autogiro como la mejor aeronave para el transporte sanitario:

- I Congreso Internacional de Aviación Sanitaria celebrado en París en 1929. En este participaron los "padres" de la aviación sanitaria y establecieron las bases de esta nueva disciplina aérea y sanitaria. En la primera ponencia L'aviation sanitaire sur les theatres d'operations extérieurs, M. le Colonel Cheutin y M. le Medecin Colonel Epaulard, nombran al autogiro como una de las aeronaves más indicadas para el transporte aéreo sanitario ${ }^{8}$. En este congreso se realiza la primera exhibición aérea mundial de un aparato de alas rotatorias para uso sanitario en el aeródromo de Villacourby el 17 de mayo de 1929 pilotado por el Sr. Massot ${ }^{(2)}$.

- II Congreso Internacional de Aviación Sanitaria celebrado en Madrid en 1933. España se suma al comienzo de esta nueva disciplina aérea y sanitaria celebrando un congreso internacional con la presencia figuras más importantes de la época. Ya en la ponencia inaugural realizada por el presidente del congreso el Dr. Cardenal nombra al autogiro como la aeronave que resuelve los problemas para el trasporte de enfermos y pide la autorización para el uso en este cometido. También en todas las siguientes comunicaciones españolas se exponen las ventajas del autogiro para el transporte sanitario. Es de resaltar la ponencia del teniente coronel Emilio Herrera director de la Escuela Superior de Aerotecnia (persona clave en el desarrollo del autogiro que llego al empleo de general y fue nombrado más tarde presidente de la republica en el exilio), que nos indica "la idea del inventor de desarrollar un modelo especifico para el trasporte sanitario" ${ }^{3,9,10}$.

- III Congreso de Aviación Sanitaria celebrado en Bruselas en 1935. Comunicación presentada por el Comandante médico Mariano Puig Quero, jefe del Servicio Sanitario de la Aviación Militar española, "Utilización del avión privado o comercial para fines sanitarios entre la Metrópoli y las Colonias". En esta ponencia el autor hace énfasis en la idoneidad del uso sanitario del autogiro en zonas que no tienen las infraestructuras tan desarrolladas como en los países occidentales ${ }^{11}$.

También se defiende al autogiro como el aparato más capaz para el transporte sanitario en el primer libro editado en España sobre la aviación sanitaria "Medicina aeronáutica y aviación sanitaria" del Dr. Box Cospedal en 1936 y en su propia tesis doctoral $^{12}$.

Distintos artículos publicados en revistas científicas aeronáuticas de la época como en la Revista Aérea de 1923 a 1929, Revista Aeronáutica desde 1932 en otros artículos de la Revista de Sanidad Militar ${ }^{13,14}$ así como en distintos diarios de tirada nacional. En todos estos se expone al público en general de la aparición de un aparato "revolucionario" que en su aplicación

\footnotetext{
(2) Diario El Imparcial. 18 de mayo de 1929. p 5. "Ante los congresistas de Aviación sanitaria vuela el autogiro La Cierva". La Vanguardia. Edición del sábado. 18 de mayo de 1929. p. 29. Los Aviones Sanitarios.
}

sanitaria soluciona el problema del transporte aéreo de enfermos y que su desarrollo corresponde a un español Juan de la Cierva. En el diario ABC a propósito de la muerte del afamado torero Ignacio Sánchez Mejía por una herida de asta de toro en 1934, en la plaza de Manzanares, se preguntan como no hay un autogiro sanitario para la rápida evacuación de este tipo de heridos en el país de su inventor ${ }^{15}$, más tarde la historia se repetirá con la muerte de "Paquirri" en 1984 en Pozoblanco. En La Voz de Madrid donde se expone un artículo la petición de autorización al autogiro para el transporte sanitario ${ }^{16}$. En Blanco y Negro donde lo comparan con extravagantes inventos de esos años como el "automóvil aéreo sanitario"17.

\section{Las primeras operaciones de salvamento y rescate con autogiros}

Desde un primer momento Juan de la Cierva piensa en un desarrollo internacional del autogiro para lo cual funda en Inglaterra "La Cierva Autogiro Company Limited". Posteriormente, asociándose con el empresario americano Harol Pitcairn funda en Estados Unidos de América la "Pitcairn-Cierva Autogiro Company". Es en Estados Unidos donde el autogiro toma un gran impulso en su desarrollo, aparte de su aplicación militar, también lo hace en el campo civil sobre todo para publicidad, fotografía, transporte de correo, prensa y transporte privado.

Durante el comienzo de los años 30 los autogiros empiezan a ser empleados para misiones de salvamento y sanitarias tanto en Estados Unidos como en Europa.

\section{En el ámbito civil en Estados Unidos}

El 7 de agosto 1932, J. Paul Lukens, participó con su autogiro PCA-2 en la búsqueda de un aparato Lockheed accidentado en las montañas de Harrisburg. Consiguió localizar y designar la posición, pero no encontró con vida a los tripulantes. En 1932 James Faulkner con un aparato de la Autogiro Company of America participó en el rescate de dos aviadores caídos en los Everglades, Miami. Los localizó y acompaño posteriormente a dos dirigibles que realizaron el rescate. En marzo de 1934 un autogiro tipo Kellett durante unas pruebas meteorológicas, localizo un aeroplano perdido socorriendo a la tripulación y transportando $174 \mathrm{~kg}$. en material de salvamento ${ }^{5}$.

\section{En el ejército y la marina}

Durante el verano de 1932 durante la segunda acción armada del ejercito americano en Nicaragua uno de los tres autogiros de experimentación PCA-2/XOP-1 fue destacado en los Marine Corps donde realizó evacuaciones sanitarias y reconocimiento ${ }^{18,19}$.

Durante 1936, en el Medical Field Service School, en Carlisle Barracas, Pensilvania, un autogiro realizó pruebas de evaluación como aparato de transporte y evacuación sanitaria durante un año ${ }^{20}$.

En Europa también tenemos constancia de la utilización del autogiro en labores sanitarias. El piloto Rolf von Bahr, en 1935 , 
de la compañía "AB Autogiro" realizó vuelos de taxi, rescate y ambulancia siendo muy popular en las estaciones de deportes de invierno de Suecia ${ }^{5,21}$. Voló unas 7.000 horas en autogiro, siendo probablemente el récord del mundo. Su aparato un C-30 (Cierva modelo 30), matrícula SE-AEA, se conserva y se puede admirar actualmente en el Tekniska Museet en Estocolmo (Figura 5). También podemos ver una filmación de la época de un rescate en montaña de este autogiro en "youtube"21 (Figura 6).

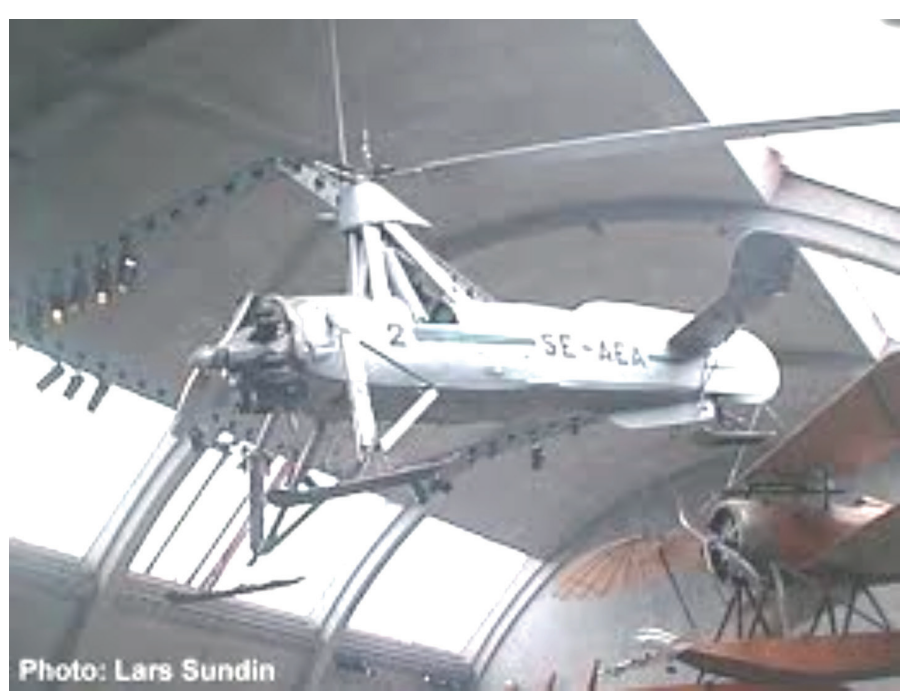

Figura 5. El autogiro de Rolf von Bahr, un C-30 matrícula SEAEA, se conserva y se puede admirar actualmente en el Tekniska Museet en Estocolmo. Este es el mismo que aparece volando en el fotograma de la figura 6 y con el que se realizaron múltiples traslados sanitarios. Imagen Lars Sundin.

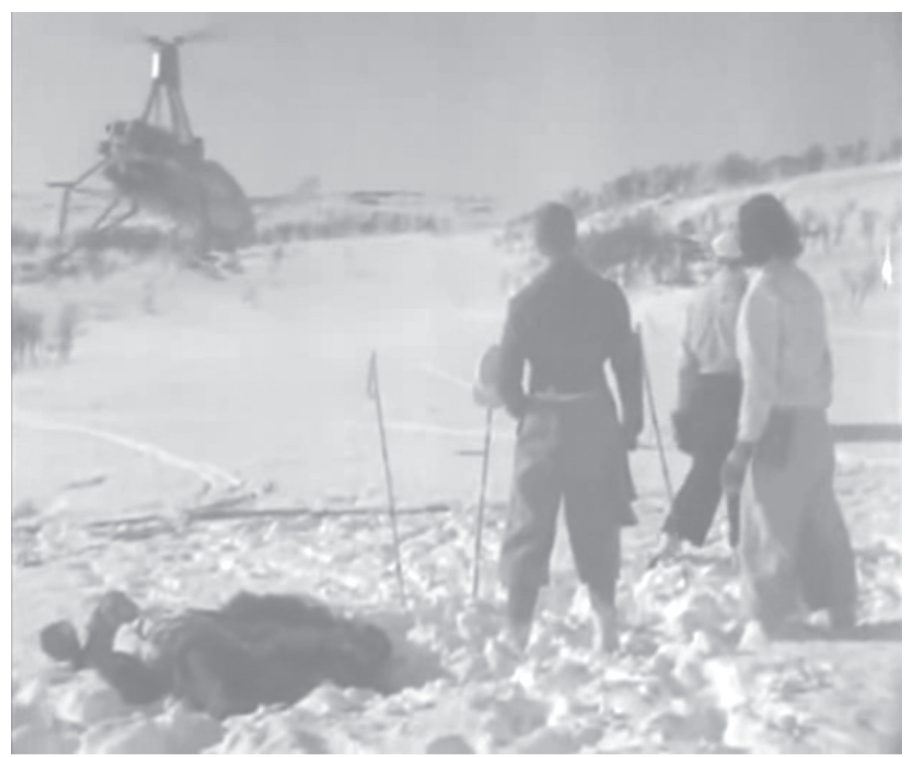

Figura 6. En la década de los 30 los autogiros fueron utilizados para el rescate y atención sanitaria tanto es así que se realizaron películas publicitarias. La imagen es de un fotograma de una película de 1938 que se puede ver en YouTube www.youtube.com/ watch? $v=I V-T 7$ Ewqt_U
En la Unión Soviética N. I. Kamov desarrolló en 1934 un autogiro A-7 con un peso de $2.000 \mathrm{~kg}$, un rotor de $15,18 \mathrm{~m}$. y una velocidad de $210 \mathrm{~km} / \mathrm{h}$, parecidos a modelos Pitcairn. Se fabricó una serie pequeña para uso militar y civil realizando misiones de salvamento en el Ártico5.

\section{El proyecto del autogiro ambulancia}

El proyecto del autogiro ambulancia se realiza en Estados - Unidos con la modificación del autogiro PA-19. Este es un autogiro diseñado por Robert B.C. Noorduyn con capacidad para trasportar cómodamente a cuatro personas y que esta propulsado por un motor Wright R-975EL. Tenía una longitud de $7,85 \mathrm{~m}$., envergadura de alas $11,79 \mathrm{~m}$. y un diámetro de rotor de $15,43 \mathrm{~m}$ y pesaba a plena carga $2105 \mathrm{~kg}$, velocidad máxima de $190 \mathrm{~km} / \mathrm{h}$ y una autonomía $560 \mathrm{~km}$. Era el mayor autogiro construido en su época. Se construyeron cuatro unidades: NC2503, NC2740, NC13149, X13182, de éstos el prototipo NC13149 fue desechado. Los dos primeros aparatos fueron comprados por el Florida Year Round Club para trasladar a cuatro pasajeros y equipaje a los hoteles y residencias de lujo de lugares turísticos de la costa americana, Miami Biltmore Country club, the Key Largo Angler`r Club y the Cabana Sun Club ${ }^{5}$ (Figura 3).

El proyecto para la transformación para uso sanitario de este robusto autogiro consistía en la modificación de las puertas de entrada al habitáculo y la instalación de soportes para dos camillas. Una posición sentada se reservaba para un sanitario o para otro pasajero. Estos mínimos cambios hacían a este autogiro el más indicado para este tipo de transporte. El propio Juan de la Cierva vuela el modelo aterrizando en el campo de fútbol americano Soldiers Field de Chicago acompañado de Harold Pitcairn cuando iban a recoger la Medalla de oro Guggenheim en la Exposición Internacional de Chicago de 1933 (Figura 7).

En junio de 1933 el Teniente Coronel Médico Dr. G. P. Lawrence publica en la prestigiosa revista "U.S. Air Services" vol XVIII. No. 6. el proyecto del autogiro ambulancia en su ar-

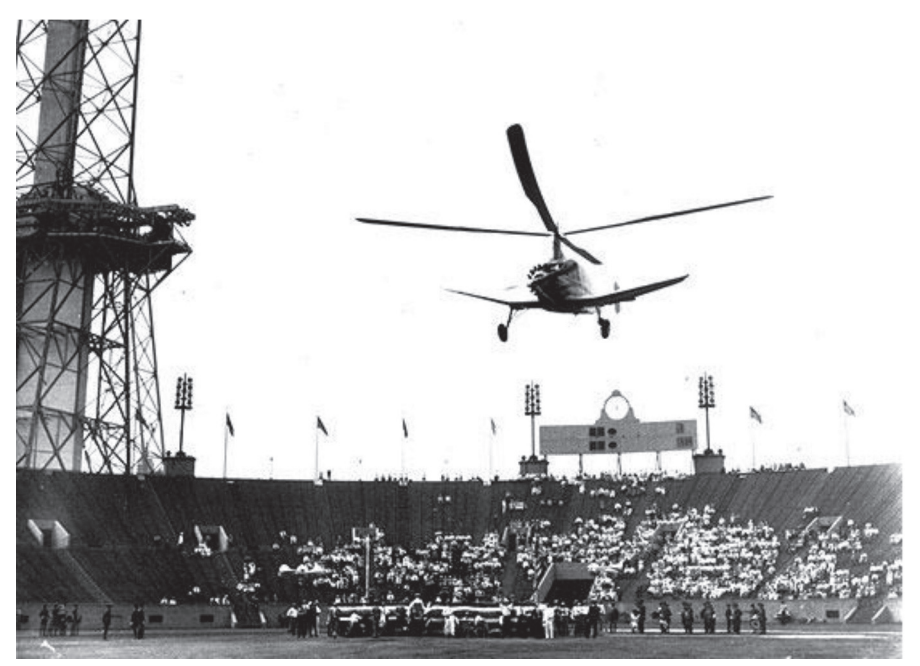

Figura 7. Aterrizaje de un PA-19 en el campo de fútbol americano Soldiers Field de Chicago con Juan de la Cierva acompañado de Harold Pitcairn. Imagen Hofstra University photo gallery. 


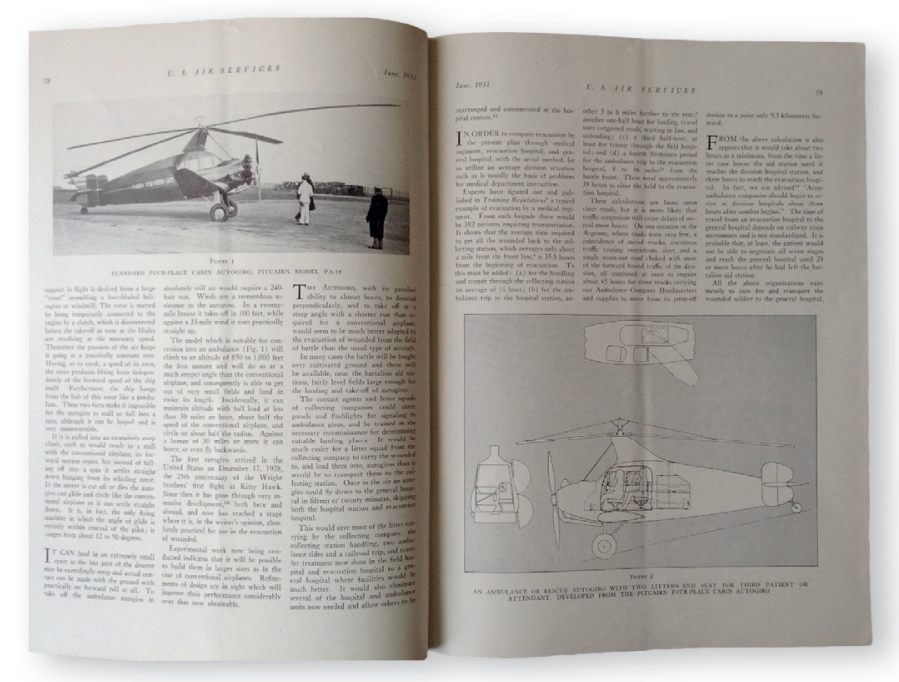

Figura 8. Revista "U.S. Air Services" vol XVIII. No. 6. de 1933 donde se refleja el proyecto del Autogiro Ambulancia en su artículo "Ambulantes take to the air" y se exponen los planos para la adaptación sanitaria. Imagen del autor.

tículo "Ambulantes take to the air", donde se refiere al autogiro como la aeronave más versátil para el transporte aéreo sanitario y como se tiene que incorporar al ejercito para satisfacer las necesidades de trasporte sanitario ${ }^{22}$ (Figura 8).

La Aviación Militar española consideró la adquisición de este modelo en versión ambulancia mediante una oferta realizada por la Pitcairn Autogiro Company al precio por aparato de 17.950 dólares f.o.b. Nueva York el día 5 de junio de $1933^{5}$. En estas fechas se estaba celebrando en Madrid el II Congreso Internacional de Aviación Sanitaria, por lo que no es raro pensar que fuera una fecha específicamente indicada para realizar la oferta, aunque no tenemos constancia de ello. Si se hubieran adquirido estos autogiros la Aviación Militar española habría sido la primera en el mundo en utilizar aparatos de ala rotatoria específicamente diseñados para el transporte sanitario, adelantándose a todas las demás naciones en este campo de la medicina y de la aeronáutica, once años antes de la conocida primera utilización del helicóptero en una misión sanitaria.

\section{El sueño de una aviación sanitaria moderna}

La gran importancia que tuvo en su época el invento del autogiro, hoy en día casi olvidado, contrasta con la poca difusión de unas de sus principales aplicaciones, el transporte sanitario. Esta aplicación ha sido una de las más importantes de los aparatos de ala rotatoria, muy por delante de las que se pensaron en un principio como la publicidad, fotografía o el transporte de periódicos y correo ${ }^{23}$.

Así como el invento y la figura de su creador fueron relegados a un segundo plano en España, en el extranjero gozaron de mayor éxito, sobre todo en EEUU.

En estos años de gran desarrollo de nuevas ideas en EEUU se presentó al autogiro como el aparato que solucionaba el transporte aéreo privado. Entre estas ideas futuristas se pensó en acercar las aeronaves hasta el propio domicilio, donde se podía tener un autogiro con el rotor plegable y apariencia de coche utilitario (autogiro Pitcairn PA 22 o 36) para uso diario. También revolucionaba el reparto de correo en las ciudades, era una plataforma perfecta para la vigilancia policial, la fotografía, la publicidad, etc. En este orden de cosas el Teniente Coronel Médico Dr. G.P. Lawrence presenta en el año 1933 al autogiro como solución real para el transporte de heridos en el ejército americano describiendo una organización de transporte sanitario a base de autogiros que acompañaría a las unidades de combate. Estos se encargarían del transporte de los heridos desde posiciones muy cercanas al frente hasta los distintos hospitales de campaña. Esta última idea es muy parecida a lo que actualmente son las unidades de aeronaves de ala rotatoria para transporte sanitario.

Todos estos distintos desarrollos del autogiro sufrieron un brusco frenazo debido a la depresión americana de los años 30 y definitivamente por la prematura muerte del inventor en accidente en 1936. Debido a esto y junto con el desarrollo posterior del helicóptero el proyecto del autogiro ambulancia fue olvidado, no existiendo actualmente constancia en los principales museos aeronáuticos americanos ni entre los propios conservadores de los archivos de Pitcairn ${ }^{(3)}$.

En nuestro país, también se intentaron desarrollar las múltiples aplicaciones del autogiro ${ }^{24}$, pero la muerte del inventor, la depresión económica de la década de los años 30 y sobre todo la problemática situación política de estos años fueron factores determinantes en el olvido de estos proyectos.

En este año 1933 se perdieron dos oportunidades de desarrollar una Aviación Sanitaria moderna. Por una parte no aplicar las diferentes recomendaciones del II Congreso Internacional de Aviación Sanitaria celebrado en Madrid y por otra, no ser el primer país en utilizar el autogiro ambulancia, antecesor del helicóptero sanitario actual.

La revolución de la aplicación del autogiro al transporte sanitario podría haber sido en el campo sanitario de la misma importancia que fue la aparición del autogiro en el campo aeronáutico.

\section{CONCLUSIONES}

El proyecto del autogiro ambulancia se publica en Estados Unidos en 1933 siendo el primer proyecto mundial de una aeronave de alas rotatorias para uso sanitario. Este se basa en la modificación para el transporte de dos enfermos tumbados del autogiro PA-19 fabricado por Pitcairn Autogiro Company, em-

\footnotetext{
(3) Correspondencia del autor con:

- Smithsonian Nacional Air and Space Museum, (Mr. Norman G. Richard, Archives Division) Washigton D.C. y Experimental Aircraft Association, Oshkosh,, Wisconsin en EEUU.

- Custodios actuales de los archivos de Pitcairn: Gregory A. Jackson, Archives \& Special Collections, Bryn Athyn Historic Landmark District, Glencairn Museum.

- Carl Gunther historiador y documentalista de Pitcairn

- Prof.Bruce Charnov autor de From Autogiro to Gyroplane: The Amazing Survival of an Aviation Technology, organizador de las jornadas en la Universidad de HOFSTRA From Autogiro to Gyroplane:The Past, Present, and Future of an Aviation Industry, 2003.
} 
presa concesionaria de las patentes del autogiro de Juan de la Cierva en EEUU.

Los médicos, ingenieros y aviadores españoles fueron los primeros defensores en foros nacionales e internacionales de la utilización de las "aeronaves de ala rotatoria" en para el transporte aéreo sanitario desde el año 1929.

El autogiro de la Cierva es el primer aparato de ala rotatoria que se utiliza en Europa y en Estados Unidos de América para misiones de salvamento y transporte sanitario. También fue el primero que realizó una exhibición pública de sus características de vuelo para el transporte sanitario en el año 1929.

España perdió la oportunidad de ser el primer país en desarrollar una Aviación Sanitaria moderna al no adquirir el primer aparato de ala rotatoria específico para el uso sanitario en el año 1933 y al no aplicar las recomendaciones del II Congreso Internacional de Aviación Sanitaria celebrado en Madrid.

\section{BIBLIOGRAFÍA}

1. Lam DM. The Aerochir: the first "flying hospital." Aviation Space Environmental Medicine 2005; 76:1174 -9.

2. González Canomanuel MA. El comienzo del transporte aéreo sanitario en España. De la campaña del norte de África al servicio de búsqueda y salvamento. Sanidad militar 2013; 69 (4): 276-282.

3. Bada Vasallo M. Aviación sanitaria. Revista de Aeronáutica 1933;16:369-373

4. Postigo JA. Juan de la Cierva y Codorniú. Ensayo para la Fundación INTEGRA. Murcia.2010.

5. Warleta Carrillo J. Autogiro: Juan de la Cierva y su obra. Madrid. Instituto de España. Cultura y Ciencia.1977.

6. Diario ABC edición Madrid. 24-2-1934. pag 28

7. Editorial. El nuevo autogiro La Cierva de mando directo y su aplicación militar. Revista Aeronáutica 1933;21:638-640.
8. Cheutin, Epaulard. Premiere question. L'aviation sanitaire sur les theatres d’operations extérieurs, Premier Congrés Internacional de l'Aviation Sanitaire.,Paris.1929.7-11.

9. Herrera E, Elices A. La seguridad de los aviones sanitarios en todas sus aplicaciones. II Congreso internacional de Aviación Sanitaria. Libro de ponencias. Madrid. Consorcio editorial.1933.24-26

10. VII Congreso de Medicina y Farmacia Militares y el de Aviación Sanitaria que acaban de verificarse en Madrid. Redacción. Revista de Sanidad Militar 1933. XXIII;6:171-172

11. Puig Quero M. Utilización del avión privado o comercial para fines sanitarios entre la Metrópoli y las Colonias. Revista de Aeronáutica 1935;41:394-398

12. Box Cospedal A. Medicina aeronáutica y aviación sanitaria. Imprenta Rosaura.Ceuta.1936.106-108

13. Escudero E. Contraindicaciones médico-quirúrgicas del transporte en aviones sanitarios. Revista de Sanidad Militar 1935.XXV; 8:259-270.

14. Sillevaerts Ch. La Aviación Sanitaria. Revista de Sanidad Militar 1930. XX;4: 97-101.

15. Diario ABC edición Madrid. G. Corrochano. Luto. 18/8/1934 pag. 15

16. Diario La Voz edición Madrid. Año XIV. Num 3880. 3/6/1933. 4

17. Blanco y Negro edición Madrid. Tom Ray. El automóvil aéreo. 21-10-1928. 105

18. Cooney C. Rotary-Wing pipeline. Edited by the staff of Naval aviation news. Naval aviation training. 75 years of Naval Aviation. Washinton DC. 1987. 50-52

19. Parker Temple L.. Of autogyros and dinosaurs. Airpower journal 1988.II.3.

20. Spurgeon H. N. Helicopter evacuation in Korea. U.S. Armed forces medical journal 1955. VI. 5

21. Henriksson L. Helicopters. Militay Aviation in Sweden. 2007. (consultado 1-8-2014). Disponiible en: http://www.avrosys.nu/aircraft/Heli/Cierva_se-aea/ CiervaSE-AEA.htm

22. Lawrence G P. Ambulances take to the air. U.S. Air Services. 1933.18.; 6:1621.

23. Martín-Barbadillo T. El autogiro. Ayer, hoy, mañana. Talleres Espasa-Calpe. Madrid. 1935.

24. Cierva J. Estudio sintético del sistema de vuelo por alas autorotativas. Memoria presentada al concurso Duque de Berwick y de Alba. Archivo de la Académia de Ciencias Exactas, Físicas y Naturales. Madrid.193 